\title{
Kidney biopsy findings in heterozygous Fabry disease females with early nephropathy
}

\author{
Carmen Valbuena • Elísio Carvalho • Manuela Bustorff • Mariana Ganhão • \\ Sandra Relvas • Rosete Nogueira • Fátima Carneiro • João Paulo Oliveira
}

Published online: 30 May 2009

(C) Springer-Verlag 2009

\section{Erratum to: Virchows Arch}

\section{DOI 10.1007/s00428-008-0653-2}

There was an error in Table 6. The error was limited to the data shown in the table and did not affect any of the statistical analyses presented in the Results and Discussion, which were based on a SPSS file that the authors have double-checked for accuracy. The corrected table is reproduced here.

The authors very much regret their error.

The online version of the original article can be found at http://dx.doi. org/10.1007/s00428-008-0653-2

C. Valbuena $\cdot$ M. Ganhão $\cdot$ S. Relvas $\cdot$ F. Carneiro

Department of Pathology, Hospital São João,

Porto, Portugal

C. Valbuena $\cdot$ F. Carneiro

Department of Pathology, Faculty of Medicine,

University of Porto,

Porto, Portugal

E. Carvalho $\cdot$ M. Bustorff $\cdot$ J. Paulo Oliveira

Department of Nephrology, Hospital São João,

Porto, Portugal

R. Nogueira

Department of Pathology, Centro Hospitalar de Gaia,

Vila Nova de Gaia, Portugal

F. Carneiro

Department of Pathology, Institute of Molecular

Pathology and Immunology (IPATIMUP),

University of Porto,

Porto, Portugal

J. Paulo Oliveira

Department of Genetics, Faculty of Medicine,

University of Porto,

Porto, Portugal

C. Valbuena $(\bowtie)$

Serviço de Anatomia Patológica, Hospital São João,

Alameda Hernâni Monteiro,

4200-319 Porto, Portugal

e-mail: valbuena@med.up.pt 
Table 6 Comparison of the three largest kidney biopsy series reporting pathology findings in females with Fabry disease

\begin{tabular}{|c|c|c|c|c|c|c|c|c|c|c|c|c|}
\hline \multirow[b]{2}{*}{ Age at kidney biopsy (years) } & \multicolumn{3}{|c|}{ Gubler et al. [10] } & \multicolumn{5}{|c|}{ Gubler et al. [10] } & \multicolumn{4}{|c|}{ Present series } \\
\hline & 8 & 22 & 51 & 30 & 37 & 54 & 55 & 73 & 32 & 42 & 45 & 50 \\
\hline CKD stage & 0 & 0 & 2 & $?$ & 2 & 4 & 5 & 4 & 2 & 1 & 2 & 2 \\
\hline Proteinuria & 0 & 0 & 0 & +++ & +++ & +++ & ++++ & ++++ & ++++ & 0 & ++ & 0 \\
\hline No. of evaluated glomeruli & 8 & 15 & 25 & 2 & 4 & 17 & 23 & 5 & 12 & 31 & 29 & 17 \\
\hline No. of sclerotic glomeruli & 0 & 0 & 5 & 1 & 2 & 17 & 22 & 3 & 9 & 2 & 7 & 4 \\
\hline (\% of sclerotic glomeruli) & $0 \%$ & $0 \%$ & $20 \%$ & $50 \%$ & $50 \%$ & $100 \%$ & $96 \%$ & $60 \%$ & $75 \%$ & $6 \%$ & $24 \%$ & $24 \%$ \\
\hline Tubular atrophy/interstitial fibrosis & 0 & 0 & + & 0 & + & ++ & +++ & + & $+/++$ & + & + & + \\
\hline
\end{tabular}

CKD stages classified according to the clinical practice guidelines of the National Kidney Foundation [33], using reported Creatinine Clearance or available algorithms to estimated GFR (eGFR): "0" — no evidence of kidney disease, "1" — eGFR $\geq 90 \mathrm{ml} / \mathrm{min} / 1.73 \mathrm{~m} 2$, "2" — eGFR = 89$60 \mathrm{ml} / \mathrm{min} / 1.73 \mathrm{~m} 2$, “3" — eGFR = 59-30 ml/min/1.73m2, “4" — eGFR = 29-15 ml/min/1.73m2, "5" — eGFR < $15 \mathrm{ml} / \mathrm{min} / 1.73 \mathrm{~m} 2$.

No. of sclerotic glomeruli is the total number of evaluated glomeruli that showed either segmental or global sclerosis.

Proteinuria was graded as: "0" $=$ no overt proteinuria, "+" $=0.3-0.5 \mathrm{~g} / \mathrm{day}$, "++" $=0.5-1.0 \mathrm{~g} / \mathrm{day}, "+++"=1.0-3.5 \mathrm{~g} / \mathrm{day}, "++++" \geq 3.5 \mathrm{~g} / \mathrm{day}$.

Tubular atrophy/interstitial fibrosis graded as: " $0 "=$ absent, "+" = mild, "++" = moderate, "+++" = severe. 